\title{
Pressão hidrostática nos atributos sensoriais do néctar de mamão
}

\author{
Hydrostatic high pressure in sensory attributes of papaya nectar
}

Fernanda Branco Shinagawa $^{\mathrm{I}^{*}}$ Rosires Deliza $^{\mathrm{II}}$ Amauri Rosenthal ${ }^{\mathrm{II}}$ Maria Alice Zarur

RESUMO

\begin{abstract}
A indústria de alimentos utiliza a análise sensorial como ferramenta essencial no desenvolvimento, otimização, controle de qualidade e análise do potencial mercadológico de novos produtos. A alta pressão hidrostática (APH) é uma tecnologia inovadora que permite manter a qualidade dos alimentos em termos nutricionais e sensoriais, bem como o frescor natural e aumentar a sua vida útil. A APH não afeta ligações covalentes, portanto são mínimos os efeitos nos constituintes químicos responsáveis pela cor, sabor e conteúdo nutricional. Este estudo teve como objetivo investigar o efeito da APH aplicada à polpa de mamão nos atributos sensoriais do néctar. Para tal, polpa controle (sem tratamento), pasteurizada $\left(92^{\circ} \mathrm{C} 40 \mathrm{~s}^{-1}\right) e$ pressurizada (300MPa $5 \mathrm{~min}^{-1} 25^{\circ} \mathrm{C}^{-1}$ ) e quatro marcas comerciais de polpa de mamão congeladas foram utilizadas para preparar os sete respectivos néctares. Estudos iniciais foram realizados para determinação da quantidade de água e açúcar "ideal" a serem adicionadas à polpa para o preparo do néctar, de acordo com a resposta dos consumidores. As sete amostras foram analisadas por 12 provadores selecionados e treinados, utilizando a Análise Descritiva Quantitativa (ADQ). Os resultados mostraram que os néctares das amostras controle e pressurizada foram similares quanto aos atributos sensoriais, tendo sido caracterizadas pela presença de grumos, sabor característico de mamão, consistência e aroma característico de mamão.
\end{abstract}

Palavras-chave: néctar de frutas, tecnologia inovadora de conservação de alimentos, análise descritiva quantitativa.

\section{ABSTRACT}

The food industry uses the sensory evaluation as an essential tool in the development, optimization, quality control and the analysis of new product potential market. High hydrostatic pressure (HHP) is an innovative technology that allows retaining the nutritional and sensory food quality, as well as the natural freshness, and extends product shelf life. The HHP does not affect covalent bonds; therefore, it has little effect on chemical constituents responsible for the color, flavor and nutritional content. This study aimed at evaluating the effect of HHP applied to the papaya pulp on sensory attributes of papaya nectar. Control (without treatment),

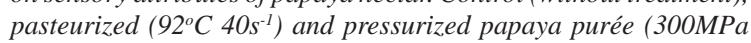
$5 \mathrm{~min}^{-1} 25^{\circ} \mathrm{C}^{-1}$ ) and four commercial papaya frozen purées available in the market were used to prepare the seven respective papaya nectars. Preliminary studies were carried out to determine the amount of water and sugar to be added to the purée, according to the consumer preference. The seven samples were evaluated by 12 selected and trained panelists using the Quantitative Descriptive Analysis (QDA). The results showed that the nectars of control and pressurized samples were similar regarding sensory attributes having been characterized by the presence of lumps, characteristic flavor of papaya, consistency and characteristic aroma of papaya.

Key words: nectars fruit, innovative technology for food preservation, quantitative descriptive analysis.

\section{INTRODUÇÃO}

Os frutos constituem parte essencial de uma dieta balanceada e são apreciados pelas excelentes propriedades sensoriais, em particular pela cor, aroma e sabor. Nesse sentido, o mamão (Carica papaya L.) se destaca como fruta de grande aceitação no mercado internacional, tanto pelo valor nutritivo como pelas qualidades sensoriais. É uma das frutas mais amplamente cultivadas nas regiões tropicais, sendo plantada na quase totalidade do território brasileiro, com destaque para a região Nordeste (RIBEIRO, 2009).

De acordo com OLSEN et al. (2010), a demanda atual por produtos industrializados é

'Escola de Química, Universidade Federal do Rio de Janeiro (UFRJ), 21941-901, Rio de Janeiro, RJ, Brasil. E-mail: fernanda.shinagawa@gmail.com.

*Autor para correspondência.

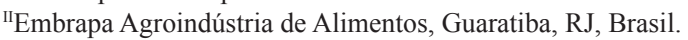


crescente, devido às mudanças no padrão de vida da população e a busca por alimentos naturais, sem adição de conservantes, que possuam qualidade e praticidade. Atualmente, o mercado de polpas de frutas congeladas tem apresentado crescimento considerável e demonstrado grande potencial mercadológico, em função da variedade de frutas disponíveis e das características em relação ao atendimento às referidas demandas (BRITO, 2011). Além disso, a industrialização de frutas tropicais pode ajudar a resolver problemas causados pelas perdas ocorridas no campo, no transporte e armazenamento, favorecendo a disponibilidade das frutas mesmo nos períodos de entressafra (FAGUNDES \& YAMANISHI, 2002).

Os processos mais comumente utilizados para a conservação de alimentos envolvem o tratamento térmico, que resulta na eliminação ou inibição de microrganismos, inativação de enzimas e de outros fatores antinutricionais, porém, ocorrendo frequentemente reações indesejáveis de natureza química que comprometem a qualidade do produto (FELLOWS, 2006).

A Alta Pressão Hidrostática (APH) é uma tecnologia de grande potencial para a indústria de alimentos por oferecer inúmeras oportunidades para o desenvolvimento de novos alimentos e, ao contrário dos tratamentos térmicos, onde tanto ligações covalentes como não-covalentes são afetadas, o processamento por alta pressão em temperatura ambiente apenas rompe ligações químicas relativamente fracas (pontes de hidrogênio, ligações hidrofóbicas e iônicas) (HENDRICKX et al., 1998), favorecendo a manutenção de compostos funcionais, da cor e do sabor do produto. Como consequência dessa nova tecnologia, surgem novos produtos, os quais precisam ser avaliados e aprovados pelo consumidor, visando a investigar a reação no mercado a fim de estimar seu sucesso ou fracasso comercial (SORENSON \& HENCHION, 2011; COSTA et al., 1999).

A indústria alimentícia vem cada vez mais utilizando a análise sensorial como ferramenta de avaliação das características sensoriais dos produtos no desenvolvimento, otimização, vida útil, controle de qualidade e avaliação do potencial de mercado de um determinado alimento (BI, 2003). Vários são os métodos empregados para avaliar as referidas características sensoriais e, dentre os quais, encontrase a Análise Descritiva Quantitativa (ADQ). Embora diversos estudos tenham investigado os efeitos da APH no processamento de distintos produtos (BOULEKOU et al., 2011), poucos enfocaram o efeito da tecnologia nas características sensoriais
(ARAYA et al., 2009; LABOISSOÈRE et al., 2007) e nada foi realizado em relação à polpa de mamão. Considerando que as características sensoriais têm relevante papel na apreciação do produto pelo consumidor, investigá-las torna-se fundamental, sobretudo quando um processo inovador é utilizado. Diante desse fato, este estudo teve como objetivo investigar o efeito da $\mathrm{APH}$ aplicada à polpa de mamão nos atributos sensoriais do néctar.

\section{MATERIAL E MÉTODOS}

Frutos de mamão cv. 'Formosa' (Carica papaya L.) provenientes de Linhares/ES foram utilizados neste estudo para o processamento de polpa. Os mamões foram mantidos em estufa de ar ventilado com controle de umidade (90\% U. R.) e temperatura $\left(10^{\circ} \mathrm{C}\right)$ até o momento do processamento. Quatro diferentes polpas pasteurizadas congeladas de marcas comerciais disponíveis no mercado do Rio de Janeiro - RJ, também foram utilizadas e mantidas a $-18^{\circ} \mathrm{C}$ até o momento da análise.

\section{MÉTODOS}

Preparo da polpa de mamão

O ponto de maturação dos frutos adotado para o processamento baseou-se no adequado teor de sólidos solúveis totais, ou seja, quando atingiram $10^{\circ}$ Brix (TEIXEIRA et al., 2001). Os frutos selecionados foram submetidos ao descascamento e retiradas as sementes para posterior despolpamento em despolpadeira (marca BONINA, mod. 025dF A8). Após o processamento, as polpas foram armazenadas em câmara de congelamento a $-18^{\circ} \mathrm{C}$ até a realização das etapas subsequentes.

Formulação do néctar de mamão

Foi escolhido para a realização da análise sensorial da polpa de mamão a formulação do néctar que melhor representava a forma em que ela é consumida. Para a formulação do néctar de mamão, foi necessário identificar a doçura e a diluição "ideal" sob o ponto de vista do consumidor. Para tal, 80 consumidores de mamão foram convidados a participar dos referidos estudos, os quais seguiram os procedimentos descritos por DELIZA (2001). Para a determinação da diluição "ideal", foram preparadas cinco diferentes concentrações de néctares de mamão $(28,33,40,50,66 \%$ de polpa de mamão), adicionadas de $7 \%$ de sacarose, concentração sugerida pelos fornecedores de polpa de mamão comercial. As amostras foram avaliadas quanto à diluição em escala 
não estruturada de nove centímetros, ancorada nos extremos com as expressões 1: "muito ralo" e 9: "muito concentrado". A partir do resultado obtido na diluição "ideal", cinco diferentes concentrações de sacarose $(4,5,6,7$ e $8 \%$ ) foram utilizadas e os néctares avaliados pelos consumidores para determinar a doçura "ideal". A escala empregada foi semelhante, variando de 1: "pouco doce" a 9: "muito doce". O ponto central das duas escalas continha a expressão "ideal" e correspondeu ao valor 5 .

Processamento térmico

A polpa de mamão foi descongelada sob refrigeração e formulada segundo doçura e diluição "ideal”. Após formulação, o néctar foi processado no binômio usualmente utilizado pela indústria de néctares no Brasil $\left(92^{\circ} \mathrm{C}\right.$ por $\left.40 \mathrm{~s}\right)$ em trocador de calor de superfície raspada (marca ARMFIELD mod. FT 25D).

Processamento por alta pressão hidrostática

Para o processamento por alta pressão, foi utilizado um equipamento em escala experimental, no qual as amostras de polpa, embaladas em sacos plásticos de polietileno de alta densidade com capacidade de $100 \mathrm{~mL}$, foram submetidas à alta pressão no equipamento Stansted fluid Power (modelo S-FL-850-9-W). O equipamento operou a uma taxa de pressurização de $100 \mathrm{MPa} \min ^{-1}$ até a pressão desejada (300MPa $\left.5 \mathrm{~min}^{-1} 25^{\circ} \mathrm{C}^{-1}\right)$.

\section{Análise descritiva quantitativa}

A análise descritiva quantitativa (ADQ) dos néctares de mamão seguiram as recomendações de STONE et al. (1974), desde o levantamento de atributos até a avaliação das amostras propriamente dito. Foram submetidas à ADQ sete amostras de néctares de mamão, formulados a partir das polpas congeladas: controle (sem tratamento), pasteurizada $\left(92^{\circ} \mathrm{C} 40 \mathrm{~s}^{-1}\right)$, pressurizada $\left(300 \mathrm{MPa} 5 \mathrm{~min}^{-1} 25^{\circ} \mathrm{C}^{-1}\right)$ e quatro marcas comerciais de polpa de mamão congeladas obtidas do mercado, a fim de possibilitar maior variedade de amostras para definição dos extremos das escalas. Todas as amostras foram formuladas segundo a diluição e a doçura "ideal", definidas e descritas anteriormente para definir os extremos das escalas, e que seriam avaliadas posteriormente.

Doze candidatos que gostavam e consumiam mamão foram recrutados com base em seu interesse e disponibilidade para participar dos testes sensoriais. Os participantes tinham experiência prévia em análise descritiva com vários néctares de frutas tropicais. Para a identificação dos atributos sensoriais relevantes, foram utilizados néctares comerciais e também amostras do presente estudo. Os provadores desenvolveram uma lista com os atributos de néctar de mamão e, ao final, uma lista consensual foi identificada, cuja definição dos referidos atributos e referências utilizadas são apresentadas na tabela 1 . Após etapa de levantamento de atributos, foram realizadas quatro sessões de treinamento com duração aproximada de 40 min cada uma delas. Em seguida, foi realizado o teste em cabine sensorial. Para tal, as amostras foram servidas à temperatura ambiente em copos plásticos brancos para avaliação dos atributos de consistência e sabor e apresentadas em erlenmeyer de vidro transparente com tampa, para avaliação dos atributos de aparência e aroma. Todas as amostras foram codificadas com números de três algarismos aleatórios. A ordem de apresentação das amostras foi balanceada para evitar erros de posição e minimizar o efeito da primeira amostra (MACFIE et al., 1989). Os néctares foram avaliados em escalas não estruturadas de $10 \mathrm{~cm}$, variando de 0 : ausente, 1: fraco até 9: forte em duplicata, durante quatro sessões em dois dias consecutivos. Biscoito de água e sal e água mineral à temperatura ambiente foram utilizados para lavar o palato entre uma amostra e outra. $\mathrm{Na}$ avaliação dos atributos de aparência e aroma, foi utilizada luz branca nas cabines individuais e, para os atributos de consistência e sabor, iluminação vermelha.

\section{Análise estatística}

Os dados para formulação "ideal" do néctar de mamão foram analisados estatisticamente por análise de regressão linear simples. Os dados provenientes da ADQ foram analisados por Análise de Variância (ANOVA), considerando provador e amostra como causas de variação e teste de Tukey com significância de $\mathrm{P} \leq 0,05$, para checar diferença entre as médias e a Análise de Componentes Principais (ACP), utilizando o programa XLSTAT (ADDINSOFT, 2007).

\section{RESULTADOS E DISCUSSÃO}

Diluição e doçura "ideal" do néctar de mamão Para a determinação da diluição "ideal" do néctar de mamão, foi considerado como "ideal" o valor 5, a partir da equação da reta obtida $\left(y=10,455 x+0,5752, R^{2}=0,965\right)$, a qual correspondeu a $42 \%$ da polpa, isto é, deveriam ser utilizadas $42 \mathrm{~g}$ de polpa $100 \mathrm{ml}^{-1}$ de água para o preparo do néctar. Já para a determinação da doçura "ideal" do néctar de mamão utilizando $42 \%$ de polpa, revelou-se 
Tabela 1 - Atributos sensoriais, definições e referências para néctar de mamão.

\begin{tabular}{|c|c|c|}
\hline Atributos & Definição & Referências \\
\hline Cor laranja característico & $\begin{array}{l}\text { Presença de cor laranja, característico de néctar } \\
\text { de mamão. }\end{array}$ & $\begin{array}{l}\text { Fraco: néctar formulado com a polpa marca M1. } \\
\text { Forte: néctar formulado com a polpa marca M2. }\end{array}$ \\
\hline Característico & Aroma característico de néctar de mamão. & $\begin{array}{l}\text { Fraco: néctar formulado com a polpa marca M2. } \\
\text { Forte: néctar formulado com a polpa controle. }\end{array}$ \\
\hline Consistência & Fluidez do néctar percebida na boca. & $\begin{array}{l}\text { Fraco: néctar formulado com a polpa marca M3. } \\
\text { Forte: néctar formulado com a polpa controle. }\end{array}$ \\
\hline Presença de Grumos & $\begin{array}{l}\text { Fibras características do mamão percebidas na } \\
\text { boca. }\end{array}$ & $\begin{array}{l}\text { Fraco: néctar formulado com a polpa M1. } \\
\text { Forte: néctar formulado com a polpa controle. }\end{array}$ \\
\hline Característico & $\begin{array}{l}\text { Presença de sabor característico de néctar de } \\
\text { mamão. }\end{array}$ & $\begin{array}{l}\text { Fraco: néctar formulado com a polpa marca M2. } \\
\text { Forte: néctar formulado com a polpa controle. }\end{array}$ \\
\hline Gosto Doce & $\begin{array}{l}\text { Percepção de doçura estimulada pelos açúcares } \\
\text { da fruta ou pela presença de sacarose. }\end{array}$ & $\begin{array}{l}\text { Fraco: néctar formulado com a polpa controle. } \\
\text { Forte: néctar formulado com a polpa marca M2. }\end{array}$ \\
\hline Sabor Cozido & $\begin{array}{l}\text { Aroma característico de mamão coccionado, } \\
\text { advindo de tratamento } \\
\text { Térmico. }\end{array}$ & $\begin{array}{l}\text { Ausente: néctar formulado da polpa controle. } \\
\text { Forte: néctar formulado da polpa marca M2. }\end{array}$ \\
\hline
\end{tabular}

necessário $6,54 \%$ de sacarose, segundo a equação $(y=68,6 x+0,516)$, cujo $R^{2}$ foi de 0,853 .

Análise descritiva quantitativa do néctar de mamão As médias dos atributos sensoriais avaliados pela equipe de provadores são mostradas na tabela 2. Foram utilizados dados de onze dos doze provadores treinados após seleção final. A seleção foi realizada através do grau de repetibilidade e das médias das triplicatas obtidas próximas aos valores extremos dos provadores treinados.

Pode-se observar que, para o atributo cor laranja característico, as amostras controle, pasteurizada e pressurizada não apresentaram diferença significativa $(\mathrm{P}>0,05)$ entre si, demonstrando que os compostos responsáveis pela cor mantiveramse inalterados após a APH nas condições empregadas. Os elevados valores observados para as amostras comerciais sugeriram que essas foram submetidas a um tratamento térmico industrial mais severo do que o utilizado no pasteurizado desse experimento. Para reforçar esse argumento, na tabela 2, pode-se também observar valores superiores para os atributos sabor cozido e gosto doce nas amostras comerciais, quando comparado às amostras controle, pasteurizado e pressurizado.

Foi observada a manutenção do atributo aroma característico do néctar de mamão nas amostras controle, pasteurizada e pressurizada, onde se verificou que as três amostras não diferiram estatisticamente $(\mathrm{P}>0,05)$ em relação ao aroma característico e alcançaram médias superiores às amostras comerciais.

$\mathrm{O}$ atributo consistência não apresentou diferença significativa $(\mathrm{P}>0,05)$ entre as amostras processadas e a controle. Diferente para as amostras comerciais que apresentaram médias significativamente $(\mathrm{P}<0,05)$ inferiores às outras mencionadas, mais uma vez sugerindo que as amostras comerciais foram provavelmente submetidas a um processamento diferente do aplicado no experimento, quando comparado ao pasteurizado.

Não houve diferença significativa $(\mathrm{P}>0,05)$ entre as amostras controle e pressurizada para o atributo presença de grumos, que apresentaram médias superiores à pasteurizada, incluindo as comerciais. Tal resultado pode sugerir que, no processamento sob alta pressão, foi mantida a propriedade presença de grumos do néctar, como observado na amostra controle, fator este positivo, por apresentar um atributo mais próximo ao natural.

Analisando os atributos de sabor, observou-se que tanto o sabor característico como o sabor cozido não diferiram $(\mathrm{P}>0,05)$ entre as amostras controle, pressurizada e pasteurizada. Entretanto, para o atributo sabor cozido, a amostra pasteurizada 
Tabela 2 - Médias*dos atributos sensoriais** das amostras de néctar de mamão avaliadas.

\begin{tabular}{|c|c|c|c|c|c|c|c|}
\hline Atributos Sensoriais & Controle & $\begin{array}{c}\text { Pasteurizado } \\
\left(92^{\circ} \mathrm{C} / 40 \mathrm{~s}\right)\end{array}$ & $\begin{array}{c}\text { Pressurizado } \\
\left(300 \mathrm{MPa} 5 \mathrm{~min}^{-1} 25^{\circ} \mathrm{C}^{-1}\right)\end{array}$ & M1 & M2 & M3 & M4 \\
\hline Cor laranja característica & $4,2^{\mathrm{de}}$ & $3,1^{\mathrm{e}}$ & $3,5^{\mathrm{e}}$ & $5,1^{\text {cd }}$ & $8,2^{\mathrm{a}}$ & $5,9^{\mathrm{bc}}$ & $7,1^{\mathrm{ab}}$ \\
\hline Aroma característico & $5,8^{\mathrm{a}}$ & $6,5^{\mathrm{a}}$ & $6,2^{\mathrm{a}}$ & $3,1^{\mathrm{b}}$ & $1,7^{\mathrm{b}}$ & $2,6^{\mathrm{b}}$ & $2,9^{\mathrm{b}}$ \\
\hline Consistência & $7,4^{\mathrm{a}}$ & $3,5^{\mathrm{c}}$ & $7,9^{\mathrm{a}}$ & $1,8^{\mathrm{d}}$ & $3,1^{\mathrm{c}}$ & $2,1^{\mathrm{cd}}$ & $4,9^{\mathrm{b}}$ \\
\hline Presença de grumos & $8,4^{\mathrm{a}}$ & $7,7^{\mathrm{a}}$ & $8,4^{\mathrm{a}}$ & $3,0^{\mathrm{cd}}$ & $1,9^{\text {de }}$ & $1,2^{\mathrm{e}}$ & $5,5^{\mathrm{b}}$ \\
\hline Sabor característico & $8,0^{\mathrm{a}}$ & $7,2^{\mathrm{a}}$ & $7,8^{\mathrm{a}}$ & $2,6^{\mathrm{c}}$ & $1,6^{\mathrm{c}}$ & $1,9^{\mathrm{c}}$ & $4,5^{\mathrm{b}}$ \\
\hline Gosto doce & $2,9^{c}$ & $4,9^{b}$ & $3,0^{\mathrm{c}}$ & $5,9^{\mathrm{b}}$ & $8,7^{\mathrm{a}}$ & $8,3^{\mathrm{a}}$ & $5,6^{\mathrm{b}}$ \\
\hline Sabor cozido & $0,6^{\mathrm{c}}$ & $2,1^{\mathrm{c}}$ & $0,6^{\mathrm{c}}$ & $3,8^{\mathrm{b}}$ & $8,6^{\mathrm{a}}$ & $7,0^{\mathrm{a}}$ & $3,8^{\mathrm{b}}$ \\
\hline
\end{tabular}

*Letras iguais na horizontal indicam que não houve diferença significativa $(\mathrm{P}>0,05)$ entre as amostras.

**Avaliados em escala não estruturada de 10cm. M1 - M4 referem-se às amostras comerciais.

apresentou média mais elevada que para as duas outras amostras, sugerindo que houve uma tendência dessa amostra apresentar maior intensidade para o referido atributo.

Para o atributo gosto doce, houve diferença significativa $(\mathrm{P}<0,05)$ entre as amostras controle e pasteurizada, devido, provavelmente, ao processamento térmico dessa amostra, a qual foi formulada antes do tratamento térmico, podendo ter ocorrido caramelização do açúcar, acentuando a percepção do gosto doce. Já a amostra submetida à APH não diferiu estatisticamente $(\mathrm{P}>0,05)$ da amostra controle, corroborando os resultados anteriores, descritos sobre a manutenção dos atributos próximo do natural, caracterizados pela amostra controle.

As amostras comerciais apresentaram as maiores médias para os atributos sabor cozido e gosto doce. Tal resultado pode ser considerado satisfatório para as amostras experimentais, considerando que esses atributos são considerados negativos nos produtos.

A Análise dos Componentes Principais (ACP) tem sido uma ferramenta muito útil para auxiliar na interpretação dos resultados da ADQ (BORGOGNONE et al.,2001). A figura 1a mostra a posição das sete amostras de néctares de mamão e a figura $1 \mathrm{~b}$, a distribuição dos atributos sensoriais no espaço definido pela primeira e segunda dimensão (PC1 e PC2, respectivamente).

Pela análise das figuras 1a e 1b, a primeira e segunda dimensão explicaram $94,97 \%$ da variância total. O componente principal 1 respondeu por $86,21 \%$, enquanto ao componente principal 2 , por $8,76 \%$.

Na figura 1a, o PC1 separou as amostras controle, pressurizada e pasteurizada das demais, evidenciando a similaridade existente entre elas, (a)

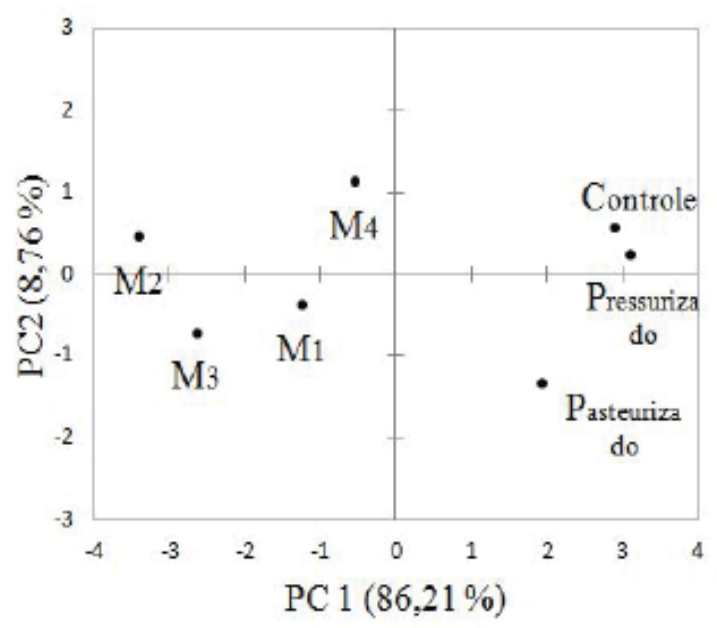

(b)

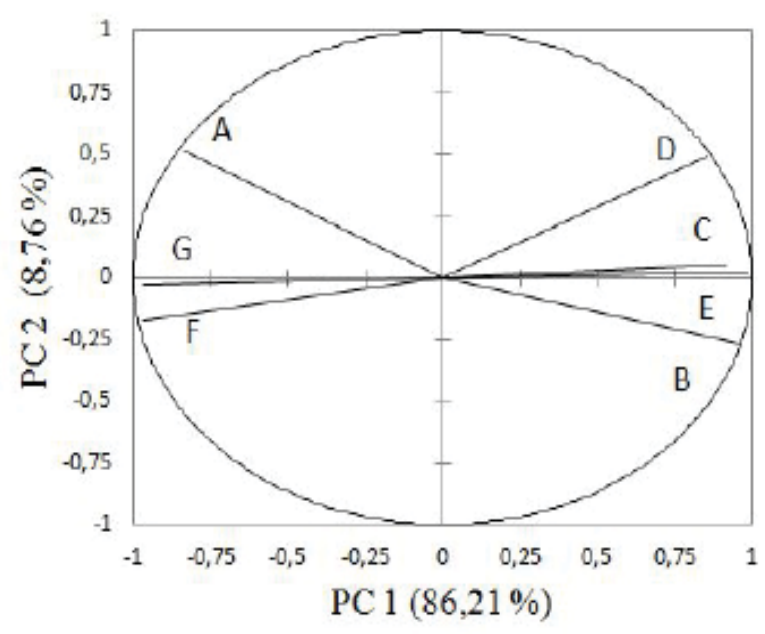

Figura 1- Análise de componentes principais (ACP) de néctar de mamão: (a) posição das amostras e (b) posição dos atributos sensoriais. 
principalmente em relação à amostra controle e a pressurizada. Na figura $1 \mathrm{~b}$, pode-se observar que os néctares dessas amostras foram caracterizados pelos atributos presença de grumos, sabor característico, consistência e aroma característico. Os das marcas comerciais de polpa de mamão foram, porém, distinguidos pelos demais atributos, como sabor cozido, cor laranja e gosto doce.

Os resultados obtidos para o néctar a partir de polpa pressurizada de mamão foram bem caracterizados em termos dos atributos sensoriais, principalmente para um produto totalmente novo que não é encontrado no mercado brasileiro. Pode-se encontrar na literatura um número elevado de estudos de análise sensorial em néctares de frutas tropicais, porém existem poucos trabalhados com os néctares pressurizados, especialmente utilizando a ferramenta da ADQ.

Dentre essas possibilidades, destacamse trabalhos de PORRETA et al. (1995), YEN \& LIN (1999), LAMBERT et al. (1999), POLYDERA et al. (2005), BARROS-MARCELLINI (2006) e LABOISSIÈRE et al. (2007), que estudaram os efeitos da APH em néctar de tomate, polpa e néctar de goiaba, compostos aromáticos presentes em polpa de morango, néctar de laranja, néctar de abacaxi e néctar de maracujá, respectivamente. Em todos esses estudos, foram comprovadas as mínimas diferenças sensoriais entre as amostras in natura com as pressurizadas, em concordância com o observado nos resultados obtidos neste trabalho.

\section{CONCLUSÃO}

Grande similaridade é verificada entre o produto obtido a partir da polpa controle e da polpa pressurizada, indicando que o processo de APH tem pouco efeito nos mesmos atributos sensoriais desses néctares, constituíndo-se de uma excelente alternativa para conservação da polpa de mamão, pois os respectivos atributos são mantidos mesmo após o processamento.

Esses resultados dão subsídios ao setor de néctares de frutas do Brasil que tem o interesse em adotar novas tecnologias para a sua conservação, a fim de manter o produto processado sensorialmente similar ao do fruto fresco, correspondendo assim à demanda dos consumidores por produtos mais próximos aos naturais.

\section{AGRADECIMENTOS}

À Fundação de Amparo à Pesquisa do Estado do Rio de Janeiro (FAPERJ) e à EMBRAPA, pelo suporte financeiro.

\section{COMITÊ DE ÉTICA E BIOSSEGURANÇA}

Processo CAAE - 0013.0.309.000-08 referente ao projeto "Conservação de polpa de frutas tropicais por alta pressão hidrostática".

\section{REFERÊNCIAS}

ADDINSOFT. XLSTAT - Pro 7.5. Excel Software. Disponível em: <http://www.xlstat.com>. Acesso em: 25 jan. 2012.

ARAYA, X.I.T. et al. Sensory perception and quality attributes of high pressure processed carrots in comparison to raw, sous-vide and cooked carrots. Innovative Food Science and Emerging Technologies, v.10, p.420-433, 2009. Disponível em: $\quad<$ http://dx.doi.org.ez67.periodicos.capes.gov.br/10.1016/j. ifset.2009.04.002>. Acesso em: 12 jan. 2013. doi: 10.1016/j. ifset.2009.04.002

BARROS-MARCELLINI, A.M. Desenvolvimento de néctar de abacaxi (Ananas comosus L. Merril) através da tecnologia de alta pressão hidrostática aplicada à polpa do fruto. 2006. 113p. Dissertação (Mestrado em Alimentos e Nutrição) - Faculdade de Engenharia de Alimentos da UNICAMP, Campinas, SP.

BI, J. Agreement and reliability assessments for performance of sensory descriptive panel. Journal of Sensory Studies, v.18, p.61-76, 2003. Disponível em: <http://onlinelibrary. wiley.com/doi/10.1111/j.1745-459X.2003.tb00373.x>. Acesso em: 28 set. 2012. doi: 10.1111/j.1745-459X.2003. tb00373.x.

BORGOGNONE, M.G. et al. Principal component analysis in sensory analysis: Covariance or correlation matrix? Food Quality and Preference, v.12, n.1, p.323-326, 2001. Disponível em: <http://www-sciencedirect-com.ez67.periodicos.capes.gov. br/science/article/pii/S0950329301000179>. Acesso em: 11 fev. 2012. doi:_10.1016/S0950-3293(01)00017-9.

BOULEKOU, S. et al. Quality evaluation of slightly concentrated tomato juice produced under high pressure conditions. Procedia Food Science, v.1, p.800-804, 2011. Disponível em: $<$ http://dx.doi. org/10.1016/j.profoo.2011.09.121>. Acesso em: 12 set. 2012. doi: 10.1016/j.profoo.2011.09.121.

BRITO, A. Projeto Pernambuco Rural sustentável PRS: relatório final de consultoria agroindústria de polpa de frutas. Recife, 2011. 29p. Disponível em: <http://www. prorural.pe.gov.br/downloads/analise_prs.pdf $>$. Acesso em: 06 jun. 2012.

COSTA, M.C. et al. Revisão: tecnologias não convencionais e o impacto no comportamento do consumidor. Boletim CEPPA, v.17, n.2, p.187-210, 1999. Disponível em: <http://ojs.c3sl.ufpr.br/ ojs2/index.php/alimentos/article/viewArticle/13791>. Acesso em: 28 set. 2012.

DELIZA, R. The use of «ideal point» scale to determine the best sugar and dilution levels of passion fruit juice by consumers. Alimentaria, v.38, n.24, p.109-113, 2001. Disponível em: <http:// dialnet.unirioja.es/servlet/articulo? codigo $=133918>$. Acesso em: 10 out. 2012. 
FAGUNDES, G.R.; YAMANISHI, O.K. Estudo da comercialização do mamão em Brasília DF. Revista Brasileira de Fruticultura, v.24, n.1, p.91-95, 2002. Disponível em: <http://www.scielo.br/ scielo.php?pid $=$ S0100-29452002000100020\&script $=$ sci arttext $>$. Acesso em: 14 jan. 2013. doi: 10.1590/S0100-29452002000100020.

FELLOWS, P.J. Tecnologia do processamento de alimentos. 2.ed. Porto Alegre: Artmed, 2006. 602p.

HENDRICKX, M. et al. Effects of High pressure on enzimes related to food quality (review). Trends in Food Science \& Technology, v.9, n.5, p.197-203, 1998. Disponível em: <http:// dx.doi.org/10.1016/S0924-2244(98)00039-9>. Acesso em: 8 mar. 2012. doi: 10.1016/S0924-2244(98)00039-9.

LABOISSOĖRE, L.H.E.S. et al. Effects of high hydrostatic pressure (HHP) on sensory characteristics of yellow passion fruit juice. Innovative Food Science and Emerging Technologies, v.8, p.469-477, 2007. Disponível em: <http://dx.doi.org.ez67. periodicos.capes.gov.br/10.1016/j.ifset.2007.04.001>. Acesso em: 11 set. 2012. doi: 10.1016/j.ifset.2007.04.001

LAMBERT, Y. et al. Changes in aromatic volatile composition of strawberry after high pressure treatment. Food Chemistry, v.67, n.1, p.7-16, 1999. Disponível em: <http://dx.doi.org.ez67. periodicos.capes.gov.br/10.1016/S0308-8146(99)00084-9> Acesso em: 28 jan. 2012. doi: 10.1016/S0308-8146(99)00084-9.

MACFIE, H.J.H. et al. Designs to balance the effects of order of presentation and first order carryover effects in hall tests. Journal of Sensory Studies, v.4, n.2, p.129-148, 1989. Disponível em: $<$ http://onlinelibrary.wiley.com/doi/10.1111/j.1745-459X.1989. tb00463.x>. Acesso em: 12 mai. 2012. doi: 10.1111/j.1745459X.1989.tb00463.x.

OLSEN, N.V. et al. Consumer acceptance of high-pressure processing and pulsed-electric field: a review. Trends in Food Science \& Technology, v.21, p.464-472, 2010. Disponível em: <http://dx.doi. org.ez67.periodicos.capes.gov.br/10.1016/j.tifs.2010.07.002>. Acesso em: 11 set. 2012. doi: 10.1016/j.tifs.2010.07.002.
POLYDERA, A.C. et al. Quality degradation kinetics of pasteurized and high pressure processed Navel orange juice: Nutritional parameters and shelf life. Innovative Food Science and Emerging Technologies, v.6, n.1, p.1-9, 2005. Disponível em: $<$ http://dx.doi. org.ez67.periodicos.capes.gov.br/10.1016/j.ifset.2004.10.004> Acesso em: 18 nov. 2012. doi:_10.1016/j.ifset.2004.10.004

PORRETA, S. et al. Effects of ultra-high hydrostatic pressure treatments on the quality of tomato juice. Food Chemistry, v.52, n.1, p.35-41, 1995. Disponível em: <http://dx.doi.org.ez67. periodicos.capes.gov.br/10.1016/0308-8146(94)P4178-I>. Acesso em: 17 out. 2012. doi: 10.1016/0308-8146(94)P4178-I.

RIBEIRO, M.L. Efeito do processamento térmico nas características físico-químicas, nutricionais, microbiológicas e na atividade enzimática de polpa de mamão Formosa (Carica papaya L.). 2009. 105p. Dissertação (Mestrado em Ciências dos Alimentos) Faculdade de Engenharia de Alimentos, Seropédica, RJ.

SORENSON, D.; HENCHION, M. Understanding consumers' cognitive structures with regard to high pressure processing: A means-end chain application to the chilled ready meals category. Food Quality and Preference, v.22, p.271-280, 2011. Disponível em: $\quad<$ http://dx.doi.org.ez67.periodicos.capes.gov.br/10.1016/j. foodqual.2010.11.003 $>$. Acesso em: 28 out. 2012. doi: 10.1016/j. foodqual.2010.11.003.

STONE, H. et al. Sensory evaluation by quantitative descriptive analysis. Food Technology, v.28, n.11, p.24-34, 1974. Disponível em: $<$ http://onlinelibrary.wiley.com/doi/10.1002/9780470385036.ch1c > Acesso em: 18 mai. 2012. doi: 10.1002/9780470385036.ch1c.

TEIXEIRA, G.H.A. et al. Processamento mínimo de mamão "Formosa". Ciência e Tecnologia de Alimentos, v.21, n.1, p.47-50, 2001. Disponível em: <http://www.scielo.br/pdf/cta/ v21n1/5363.pdf>. Acesso em: 28 set. 2012.

YEN, G.C.; LIN, H.T. Changes in volatile flavor components of guava juice with high pressure treatment and heat processing and during storage. Journal of Agriculture and Food Chemistry, v.47, n.1, p.2082-2087, 1999. Disponível em: <http://pubs.acs. org/doi/abs/10.1021/jf9810057>. Acesso em: 14 jun. 2012. doi: $10.1021 /$ jf9810057. 